\title{
Ribonuclear inclusions as biomarker of myotonic dystrophy type 2, even in improperly frozen or defrozen skeletal muscle biopsies
}

\author{
R. Cardani, ${ }^{1}$ E. Mancinelli, ${ }^{1}$ M. Giagnacovo, ${ }^{1}$ V. Sansone, ${ }^{2}$ G. Meola ${ }^{2}$ \\ ${ }^{1}$ Department of Molecular Biology and Biotechnologies, University of Milan; ${ }^{2}$ Department of Neurology, \\ IRCCS Policlinico San Donato, University of Milan, Italy
}

(C)2009 European Journal of Histochemistry

Myotonic dystrophy type 2 (DM2) is a dominantly inherited disorder caused by a CCTG repeat expansion in intron 1 of ZNF9 gene. The size and the somatic instability of DM2 expansion complicate the molecular diagnosis of DM2. In situ hybridization represents a rapid and sensitive method to obtain a definitive diagnosis in few hours, since it allows the direct visualization of the mutant mRNA foci on skeletal muscle sections. This approach makes the muscle biopsy an important tool for definitive diagnosis of DM2. Consequently, a rapid freezing at ultra cold temperature and a good storage of muscle specimens are essential to avoid morphologic alterations and nucleic acids degradation. However incorrect freezing or thawing may accidentally occur. In this work we report that fluorescence in situ hybridization may be applied on improperly frozen or inappropriately stored muscle biopsies since foci of mutant mRNA are well preserved and can still be detected in muscle sections no more useful for histopathological evaluation.

Key words: myotonic dystrophy type 2; defrozen muscle biopsy; fluorescence in situ hybridization; ribonuclear inclusions.

\section{Correspondence: Giovanni Meola,}

Professor and Chair of Neurology, Dept. of Neurology, IRCCS Policlinico San Donato University of Milan,

via Morandi 30, 20097 San Donato Milanese, Milan, Italy

Tel.: +39.02.52774480.

Fax. +39.02.5274717.

E-mail: giovanni.meola@unimi.it

Paper accepted on 23 May 2009

European Journal of Histochemistry

2009; vol. 53 issue 2 (April-June): 107-112
$\mathrm{M}$ yotonic dystrophy type 2 (DM2) is a neuromuscular disorder due to the unstable (CCTG)n repeat expansion in intron 1 of the zinc finger protein 9 ( ZNF9) gene on chromosome 3q21.3 (Liquori et al. 2001). Mutant ZNF9 pre-mRNA is spliced and polyadenylated, and the mRNA is exported to the cytoplasm where normal levels of ZNF9 protein expression occur (Botta et al., 2006; Margolis et al. 2006); however, the expanded repeats remain in cell nuclei as ribonuclear inclusions (Liquori et al. 2001). The DM2 ribonuclear inclusions contain only the CCUG repeat sequence derived from intron 1 but with no detectable flanking intronic RNA (Margolis et al. 2006). CCUG-containing mutant mRNAs form double-stranded hairpin loop structures that bind specific RNA-binding proteins such as muscleblind-like proteins (MBNLs) that colocalize with ribonuclear inclusions in myonuclei (Mankodi et al., 2001; Fardaei et al., 2002). Sequestration of these proteins which are regulators of alternative splicing, alters the splicing of several pre-mRNA (reviewed by Osborne and Thornton, 2006) such as the insulin receptor (IR) and the chloride channel (CIC1) (Savkur et al., 2004; Charlet et al., 2002; Mankodi et al., 2002). Alterations in IR splicing leads to insulin insensitivity and predisposition to diabetes (Savkur et al. 2004) and alterations in $\mathrm{CICl}$ splicing results in electrical myotonia (Charlet et al., 2002; Mankodi et al., 2002). Conventional Southern blot analysis is not adequate for a definitive molecular diagnosis in DM2 due to the extremely large size and somatic instability of the expansion mutation (Liquori et al., 2001; Bachinski et al., 2003). The extraordinary somatic instability complicates the analysis of genotype-phenotype correlations including those in the effect of the gender of transmitting parents and anticipation. The copy number of DM2 CCTG is below 30 in phenotypically normal individuals and up 11.000 in patients (Day and Ranum, 2005). A complex genotyping diagnostic proce- 
dure is now commonly used consisting of a threestep molecular protocol (Day et al., 2003; Udd et al., 2003). However, a more practical tool to obtain a definitive diagnosis in few hours is represented by in situ hybridization which detects ribonuclear inclusions in cell nuclei of muscle fibers (Cardani et al., 2004; Sallinen et al., 2004). This approach makes muscle biopsy an essential tool for DM2 diagnosis. For this reason muscle specimens should be sent fresh, for rapid freezing, from the operating room to the pathology laboratory. To avoid RNA degradation, biopsies require special precautions with handling of material, such as immediate freezing of fresh tissues, because retrospective genetic analysis is impaired by conventional tissue processing techniques. However, many small hospitals are ill-equipped for snap freezing which requires access to liquid nitrogen or dry ice; thus, frequently outside hospitals provide specimens that are obscured with freeze artefacts because they either were submitted incorrectly or were improperly frozen, at the point of origin prior to shipment. Moreover, an accidental tissue thawing and refreezing may occur (for example power failure of the freezer) causing severe tissue damages and possible RNA degradation.

Here we report our experience on DM2 muscle biopsies improperly preserved: these were no more useful for a histopathological analysis since they showed evident morphologic artefacts, but they proved to be still suitable for diagnosis by fluorescence in situ hybridization (FISH) since ribonuclear inclusions were preserved and still detectable on muscle sections.

\section{Materials and Methods}

Incorrectly frozen or accidentally thawed and refrozen muscle biopsies of biceps brachii or vastus lateralis from genetically confirmed DM2 patients $(n=11)$ have been revaluated by fluorescence in situ hybridization (FISH) analysis. Well preserved muscle biopsies from DM2 or healthy subjects were used as control $(n=5)$.

The histological diagnosis was performed on serial sections of muscle biopsies $(n=10)$ which did not underwent accidental thawing and were processed for routine histological or histochemical staining. A standard myofibrillar ATPase staining protocol was used after preincubation at pH 4.31, pH 4.61 and pH 9.4 or 10.4 (Dubowitz, 1985). Antibodies against different myosin heavy chain ( M HC) isotypes were used for immunohistochemical identification of fast and slow $\mathrm{MHC}$ fibers according to Vihola et al. (2003). Quantitative evaluation of fiber diameter was made with Scion Image (Scion Corporation, Frederick, MD) as previously reported (Vihola et. al., 2003). Relative atrophy factor was also calculated (Dubowitz, 1985). The genetic confirmation of DM2 was performed by repeat assay described by Bachinski et al. (2003).

Fluorescence in situ hybridization (FISH) was performed on $5 \mu \mathrm{m}$ thick muscle cryosections using a Texas red labelled (CAGG) 5 probe (IDT, Coralville, IA) as previously reported by Cardani et al. (2004). Briefly, transverse muscle cryostat sections were air-dried for $30 \mathrm{~min}$ at room temperature and fixed with $2 \%$ paraformaldehyde for $30 \mathrm{~min}$ at $4^{\circ} \mathrm{C}$. After washing in phosphate buffered saline (PBS). Sections were permeabilized in $2 \%$ acetone in PBS (prechilled at $-20^{\circ} \mathrm{C}$ ) for 5 min. After washing in PBS, sections were incubated in $30 \%$ formamide and 2 XSSC for 10 $\mathrm{min}$ at room temperature and then hybridized with the probe $(1 \mathrm{ng} / \mu \mathrm{L})$ for $2 \mathrm{~h}$ at $37^{\circ} \mathrm{C}$ in $30 \%$ formamide, $2 \times S S C, 0.02 \%$ BSA, $67 \mathrm{ng} / \mu \mathrm{L}$ yeast tRNA, 2 mM vanadyl ribonucleoside complex. The sections were washed first in 30\% formamide and $2 \times S S C$ at $45^{\circ} \mathrm{C}$ for $30 \mathrm{~min}$, then in IxSSC containing 165 nM 4,6 diamidino-2-phenylindole (DAPI) at room temperature for $30 \mathrm{~min}$, and finally mounted with ProLong (Molecular Probes). The muscle sections were examined with a Leica DM RA2fluorescence microscope, under the appropriate conditions of excitation and emission.

Haematoxylin and eosin staining was performed on serial sections to verify the morphology of the muscle tissue.

\section{Results}

The histopathological analysis performed on muscle sections obtained from biopsies before the accidental thawing showed the DM2 characteristic myopathologic alterations (Table 1 ). Increased fiber size variation, predominant type 2 fiber atrophy and central nucleation, nuclear clumps expressing almost exclusively fast myosin, were 
observed in all DM2 muscle biopsies examined (Figure 1A). One muscle biopsy, sent from an outside hospital, has been improperly frozen at the point of origin prior to shipment. Muscle tissue showed so many freezing artefacts that a histological or immunohistochemical analysis was not possible (Figure 1E).

The application of FISH using the fluorescentlylabelled antisense oligonucleotide (CAGG) 5 probe on section of well-frozen muscle biopsies obtained from genetically confirmed DM2 patients showed the presence in myonuclei of intense hybridization signals which represent foci of CCUG-containing RNA (Figure 1B). In this study, we have performed FISH on muscle sections obtained from DM2 biopsies with evident morphological alterations due to incorrect freezing or storage. We have also re-examined old DM2 biopsies, previously diagnosed by FISH, that underwent accidental tissue thawing and refreezing. In these biopsies tissue morphology is completely altered and artefacts may be so severe to make the available material no more useful for further analysis (Figure 1C). However the application of FISH on these muscle sections showed that mutant RNA foci can still be detected in myonuclei (Figure 1D). No aspecific signals are present in these muscle sections both in nuclei or cytoplasm, and the hybridization results were similar as those observed in properly-frozen sections.

Frequently, outside hospitals send us muscle biopsies obtained from suspected DM2 patients to perform a definitive diagnosis. Sometimes, these biopsies are either submitted incorrectly or were incorrectly frozen at the point of origin prior to shipment; thus, they show evident freezing arte-

Table 1. Histopathologic alterations in DM2 muscle.

\begin{tabular}{lcccc}
\hline Patient & \multicolumn{2}{c}{$\begin{array}{c}\text { relative atrophy } \\
\text { factor }\end{array}$} & $\begin{array}{c}\text { type } 2 \text { fiber } \\
\text { central nucleation }\end{array}$ & nuclear clumps \\
& Type 1 fibers & Type 2 fibers & & \\
\hline BC & 0,65 & 7,04 & $+^{+}$ & ++ \\
Cl & 0,30 & 8,37 & + & ++ \\
CS & 0,29 & 3,21 & + & ++ \\
CC & 3,37 & 6,95 & + & ++ \\
DD & 1,55 & 0,63 & \pm & \pm \\
LR & 0,40 & 1,12 & + & + \\
MG & 1,06 & 8,05 & + & ++ \\
NG & 1,58 & 5,65 & + & ++ \\
RO & 0,00 & 1,71 & \pm & \pm \\
Ram & 0,58 & 5,31 & + & ++
\end{tabular}

apresence of type 2 fiber central nucleation or nuclear clumps is graded as 0 absent, \pm rare, + moderate, ++ numerous; relative atrophy factor n.v. up to 1. facts which make a morphological evaluation of these muscle tissues impossible (Figure 1E). In any case, a genetic analysis of these muscle biopsies can be carried out. The application of FISH on genetically confirmed DM2 patients revealed the presence of ribonuclear inclusions in myonuclei of improperly preserved muscle sections, allowing us to confirm the DM2 diagnosis (Figure $I F$ ). No hybridization signals were present on similar muscle sections from patients whose genetic analysis had not confirmed the DM2 clinical diagnosis (Figures $1 \mathrm{G}, \mathrm{H}$ ).

\section{Discussion}

Muscle biopsy has an important diagnostic role in many neuromuscular diseases. Since it is an invasive investigation, it is important that the biopsy procedure and subsequent laboratory processing of the specimen are well performed to obtain an optimal sample for diagnosis. Ideally the specimen should be frozen immediately after excision, and the correct methods of freezing as well as the storage of frozen biopsies are crucial for the histopathological or biomolecular diagnosis (Meola, 2005). Indeed, a slow tissue freezing can yield a riddled sample as a consequence of ice crystal artefacts formation, while thawing and refreezing definitely compromise the analysis of enzymatic activities. Moreover, slow freezing or thawing-refreezing can results in DNA depletion and RNA degradation.

Histopathological evaluation of muscle biopsy appears to be of particular importance for the definitive diagnosis of DM2 pathology. Indeed, this pathology has been recently defined as "a disease of type 2 fibers" since both fiber atrophy and central nucleation selectively affect type 2 fibers (Bassez et al., 2008). It appears that variation in normal fiber size, fiber type differentiation and the nucleus position can be easily recognized in DM2 muscle sections. Moreover, muscle biopsy represents an essential tool for a definitive biomolecular diagnosis of DM2 which may be obtained in few hours by in situ hybridization which allows a direct visualization of ribonuclear inclusions on muscle sections (Cardani et al., 2004; Sallinen et al., 2004).

Our retrospective study on improperly frozen or defrozen DM2 biopsies demonstrates that ribonu- 

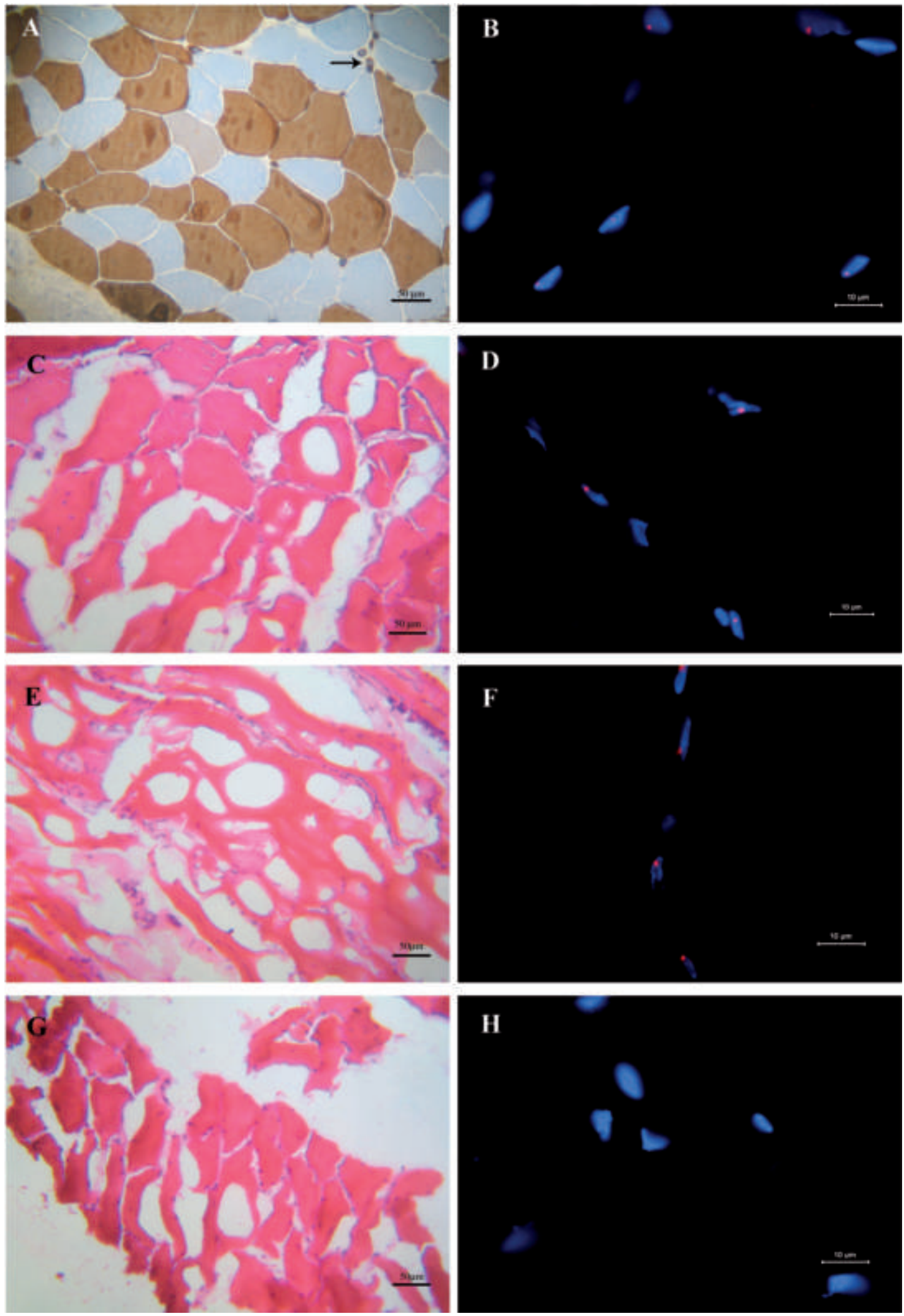

Figure 1. (A) Immunohistochemical staining of fast MHC performed on muscle section obtained from a DM2 biopsy before accidental defrozen (same patient of 1C and 1D). Black arrow indicates nuclear clumps which express the MHC fast isotype. (B) FISH with the (CAGG)5 probe on a muscle section obtained from a well frozen DM2 biopsy: ribonuclear inclusions (red spots) are present in the myonuclei (blue). C-H. Haematoxylin and eosin staining (C, E, G) and FISH (D, F, H) performed on muscle sections from DM2 biopsies which underwent accidental tissue thawing and refreezing (C, D) or were incorrectly submitted (E, F), or performed on sections of an incorrectly frozen control biopsy (G, H). In these biopsies, tissue morphology is completely altered; however ribonuclear inclusions are well detectable in DM2 muscle sections (red spots in D, F) whereas they are not present in the control section (H). 
clear inclusions, which represent an important biomarker of this pathology, are well preserved and still detectable by FISH on muscle sections. This result indicates that ribonuclear inclusions are stable and resistant to potential degradation caused by incorrect method of freezing or by thawing. Consistently, we have recently demonstrated by FISH the presence of CUG-containing RNA foci in the nuclei of gallbladder smooth muscle cells from myotonic dystrophy type 1 (DMI) patients (Cardani et al., 2008), although no special precautions had been taken to avoid RNA degradation by RNAse.

Studies carried out on DMI ribonuclear inclusions suggest that these foci are complex structures made up of mutant mRNA, RNA-binding proteins and transcription factors that physically interact each other playing an important role in the maintenance of foci integrity (Dansinthong et al., 2005; Ebralidze et al., 2004). It seems that MBNLI binding to mutant RNA plays an essential role in ribonuclear inclusions formations and maintenance in DMI where this protein also colocalizes with CUG-containing RNA nuclear foci (Dansithong et al., 2005). The stability of mutant RNA is also demonstrated by their resistance to the treatments used for formalin fixation and paraffin embedding. Indeed, Sallinen et al. (2004) demonstrates that in situ hybridization can be applied on DM2 paraffin-embedded muscle biopsy where ribonuclear inclusions are well detectable.

Our results also demonstrate that even in the presence of a biopsy which had been improperly frozen, defrozen or incorrectly submitted from other hospitals, a second biopsy might not be necessary to obtain a definitive DM2 diagnosis. On this kind of material, retrospective studies can also be conducted to screen patients with illdefined myotonic disorders.

\section{References}

Bachinski LL, Udd B, Meola G, Sansone V, Bassez G, Eymard B, et al. Confirmation of the type 2 myotonic dystrophy (CCTG)n expansion mutation in patients with proximal myotonic myopathy/proximal myotonic dystrophy of different European origins: a single shared haplotype indicates an ancestral founder effect. Am J Hum Gene
$2003 ; 73: 835-48$

Bassez G, Chapoy E, Bastuji-Garin S, Radvanyi-Hoffman H, Authier FJ, Pellissier JF et al. Type 2 myotonic dystrophy can be predicted by the combination of type 2 muscle fiber central nucleation and scattered atrophy. J Neuropathol Exp Neurol 2008; 4:319-25.

Botta A, Caldarola S, Vallo L, Bonifazi E, Fruci D, Gullotta F, et al. Effect of the (CCTG)n repeat expansion on ZNF9 expression in myotonic dystrophy type II (DM2). Biochim Biophys Acta 2006; 1762:329-34.

Cardani $R$, Mancinelli $E$, Sansone $V$, Rotondo $G$, Meola $G$. Biomolecular identification of (CCTG)n mutation in myotonic dystrophy type 2 (DM2) by FISH on muscle biopsy. Eur $\mathrm{J}$ Histochem $2004 ; 48: 437-42$

Cardani R, Mancinelli E, Saino G, Bonavina L, Meola G. A putative role of ribonuclear inclusions in the impairment of gallbladder smooth muscle contractility with cholelithiasis in myotonic dystrophy type 1. Neuromuscul Disord 2008; 18:641-5.

Charlet-B N, Savkur RS, Singh G, Philips AV, Grice EA, Cooper TA. Loss of the muscle-specific chloride channel in type 1 myotonic dystrophy due to misregulated alternative splicing. Mol Cell 2002; 10:45-53.

Dansithong W, Paul S, Comai L, Reddy S. MBNL1 is the primary determinant of focus formation and aberrant insulin receptor in DM1. J Biol Chem 2005;280:5773-80.

Day JW, Ranum LPW. RNA pathogeness of the myotonic dystrophies. Neuromuscul Disord 2005; 15:5-16.

Dubowitz V. Muscle biopsy: a practical approach. Bailliere Tindall, London 1985;19-40.

Ebralidze A, Wang Y, Petkova V, Ebralidse K, Junghans RP. RNA leaching of transcription factors disrupts transcription in myotonic dystrophy. Science 2004; 303:383-87.

Fardaei M, Rogers MT, Thorpe H M, Larkin K, Hamshere MG, Harper $P S$, et al. Three proteins, MBNL, MBLL and M BXL, co-localize in vivo with nuclear foci of expanded repeat transcripts in $\mathrm{DMl}$ and DM2 cells. Hum Mol Genet 2002; 11:805-14.

Liquori CL, Ricker K, Moseley M L, Jacobsen JF, Kress W, Naylor SL, et al. Myotonic dystrophy type 2 caused by a CCTG expansion in intron 1 of ZNF9. Science 2001;293:816-17.

Mankodi A, Urbinati CR, Yuan QP, Moxley RT, Sansone V, Krym M, et al. Muscleblind localizes to nuclear foci of aberrant RNA in myotonic dystrophy types 1 and 2. Hum Mol Genet 2001;10:2165-70.

Mankodi A, Takahashi MP, Jiang H, Beck CL, Bowers WJ Moxley RT, et al. Expanded CUG repeats trigger aberrant splicing of CIC-1 chloride channel pre-mRNA and hyperexcitability of skeletal muscle in myotonic dystrophy. Mol Cell 2002;10:35-44.

Margolis JM, Schoser BG, Moseley ML, Day JW, Ranum LPW. DM2 intronic expansions: evidence for CCUG accumulation without flanking sequence or effects on ZFN9 mRNA processing or protein expression. Hum Mol Genet 2006;15:1808-15

Meola G. Advanced microscopic and histochemical techniques: diagnostic tools in the molecular era of myology. Eur J Histochem 2005; 49:93-6.

Osborne J, Thornton CA. RNA-dominant diseases. Hum Mol Genet 2006;15:R162-R169.

Sallinen R, Vihola A, Bachinski LL, Huoponen K, Haapasalo $H$, Hackman $P$, et al. New methods for molecular diagnosis and demonstration of the (CCTG)n mutation in myotonic dystrophy type 2 (DM2). Neuromuscul Disord 2004;14:274-83.

Savkur RS, Philips AV, Cooper TA, Dalton JC, Moseley M L, Ranum $\mathrm{LPW}$, et al. Insulin receptor splicing alteration in myotonic dystrophy type 2. Am J Hum Genet 2004; 74:1309-13.

Udd B, Meola G, Krahe R, Thornton C, Ranum L, Day J, et al. Report of the 115th ENMC workshop: DM2/PROMM and other myotonic dystrophies. Neuromuscul Disord 2003;13:589-96.

Vihola A, Bassez G, Meola G, Zhang SI Haapasalo H, Paetau A, et al. Histopathological differences of myotonic dystrophy type 1 (DM1) and PROMM/DM2. Neurology 2003;60:1854-57. 
R. Cardani et al. 